\title{
Performance Evaluation of Windowing Based Energy Detector in Multipath and Multi-Signal Scenarios
}

\author{
Johanna Vartiainen ${ }^{10000-0002-0819-09243]}$, Heikki \\ Karvonen $^{1[0000-0002-1491-0422]}$, Marja Matinmikko-Blue ${ }^{1[0000-0002-0094-6344]}$, \\ and Luciano Mendes ${ }^{2}$ [0000-0002-1996-7292] \\ 1 Centre for Wireless Communications, University of Oulu, Finland \\ firstname.lastname@oulu.fi \\ 2 Radiocommunications Research Center, Inatel, Brazil \\ luciano@inatel.br
}

\begin{abstract}
Connectivity in remote areas continues to be a major challenge despite of the evolution of cellular technology. 5th Generation (5G) technology can address remote connectivity if lower carrier frequencies are available, which calls for shared use of spectrum to enable costefficient license-free solution. Therefore, spectrum sensing has its own role in future wireless systems such as mobile $5 \mathrm{G}$ networks and Internet of Things (IoT) to complement database approach in dynamic spectrum utilization. In this paper, a windowing based (WIBA) blind spectrum sensing method is studied. Its performance is compared to the localization algorithm based on double-thresholding (LAD) detection method. Both the methods are based on energy detection and can be used in any frequency range as well as for detecting all kind of relatively narrowband signals. Probability of detection, relative mean square error for the bandwidth estimation, and the number of detected signals were evaluated, including multipath and multi-signal scenarios. The simulation results show that the WIBA method is very suitable for future $5 \mathrm{G}$ applications especially for remote area connectivity, due to its good detection performance in low signal-to-noise ratio (SNR) areas with low complexity and reasonable costs. The simulation results also show importance of the used detection window selection since too wide detection window degrades the detection performance of the WIBA method.
\end{abstract}

Keywords: signal detection · spectrum utilization · $5 \mathrm{G}$ system · overlapping $\cdot$ sampling.

\section{Introduction}

5th Generation $(5 \mathrm{G})$ technology can be considered to be an extensive revolution of the mobile communication systems that brings a whole new era to connectivity. Near-future 5G system brings spectrum efficiency, scalability, intelligence, low latency and advanced security features. It enables Internet of Things (IoT) 
[1] which connects massive number of objects like computers, services and devices like sensors and mobile phones together. At the same time, it has potential to serve currently underserved remote areas. As the amount of disposable communication radio channels is limited, the effectiveness of the use of radio spectrum must be optimized. $5 \mathrm{G}$ system includes the use of higher frequencies (e.g. 3.5 and $28 \mathrm{GHz}$ ) as well as aggregated use of licensed and unlicensed bands. Traditionally, in cellular systems wireless spectrum is made available through an inflexible spectrum allocation, where frequency bands are permanently allocated to some licensed (primary) users (PU). 5G-RANGE project [13] proposes that a cost-efficient remote and rural area connection can be enabled by using $5 \mathrm{G}$ cognitive radio (CR) networks where unlicensed (secondary) users (SU) are able to use temporarily unused frequency bands (i.e. frequency holes) in licensed frequency channels leading to increased spectrum efficiency. The main requirement for shared frequency use is that the licensed user must not be interfered by $\mathrm{SU}(\mathrm{s})$. Two high-level approaches are used for that purpose: database and spectrum sensing. As databases collect and store information about licensed users such as TV and program making and special events (PMSE) signals (e.g. wireless microphone signals) in some geographical area, spectrum sensing can be used to find out (detect) which frequency bands are available for a transmission by observing the radio environment.

In $5 \mathrm{G}$ scenarios, spectrum sensing can be used to enhance the traditional database approach by bringing more accurate information about the actual spectrum usage and thus increase the potential and reliability of shared spectrum access. 5G communication application areas for spectrum sensing include, e.g., mobile cellular systems [2], device-to-device (D2D) communication [3], and IoT [4]. Sensing can be used when the information in database or from geolocation method (like GPS) is inaccurate, or there is no connection to the database at all, like in disaster-related events or in remote areas. In addition, secondary users (SU) may use sensing when defining are there other SUs present [5]. 5G can be tailored to be used for remote area connectivity where the use of TV white spaces (TVWS), i.e., Very High Frequency (VHF) and Ultra High Frequency (UHF) bands, with database can be enhanced with spectrum sensing. In rural and remote areas the challenge is that distances are long and, thus, signal-to-noise ratio (SNR) levels are low.

As $5 \mathrm{G}$ systems will include a huge number of devices, especially in IoT scenarios, design complexity and costs should stay in a reasonable level. Energy detection (ED) is a cost-efficient sensing technique that is recommended to be used especially in cooperative sensing, where users collaborate and exchange their sensing information [6]. 5G cooperative sensing based on ED methods has been studied, for example, in [7]. The problem is that conventional ED does not perform well at low SNR values.

In this paper, a novel ED-based spectrum sensing method is studied, namely the windowing based (WIBA) signal detection method [8]. This recently proposed method is an efficient blind spectrum sensing method that is able to iteratively estimate the noise level by using adaptive thresholds. The WIBA method 
uses overlapping blocks in spectrum sampling to increase its detection performance. In [8], where the WIBA method was proposed, only the probability of detection and the number of detected signals in one-signal case were studied. In this paper, the method is studied more comprehensively. The effect of the detection window length $M$ to the detection performance in different channel situations is studied. Relative mean squared error (RMSE) for the bandwidth estimation is considered, as well as detection probability over multipath channels. In addition, multi-signal situation is considered. The results are compared with the ones achieved by the widely studied localization algorithm based on double-thresholding (LAD) method [9], which has been found to outperform conventional ED methods [10].

\section{System model}

Connectivity in rural and remote areas is a true challenge because most of today's technologies aim for coverage below $10 \mathrm{~km}$ radius. In a sparsely populated area, a $10 \mathrm{~km}$ cell will only cover a small number of subscribers, resulting in very high fees per user. Another problem for realizing remote connectivity is the high cost of the spectrum licenses, which increases the investments to deploy a mobile network and hinders its economic feasibility. $5 \mathrm{G}$ in remote areas requires the use of lower frequency bands to reach wider area coverage, e.g., 50-100 km. Therefore, the upcoming $5 \mathrm{G}$ millimeterwave bands are not the first target for remote area connectivity. Instead, the use of so called TV white spaces has the potential to be used for $5 \mathrm{G}$ networks, to provide cost-efficient solution in remote areas, after its main boom over a decade ago. Administrations have developed rules for the use of TVWS and selected geolocation database approach as the means to protect the incumbent TV broadcasting usage, see e.g. [11]. In these approaches, devices wishing to access the TVWS need to inquire a database and report their location to be allowed to use a channel such that the incumbents are protected. The Federal Communications Commission (FCC) defined Citizen Broadband Radio Service (CBRS) for shared commercial use of the $3.5 \mathrm{GHz}$ band with the incumbent military radars and fixed satellite stations [12]. CBRS system includes the use of spectrum sensing in conjunction with database to avoid unlicensed users interference to military radar systems. The use of spectrum sensing to complement database approach in TVWS has been studied to some extent but has not been adopted by other system so far.

The opportunistic use of the TVWS demands protection of the incumbents. While several standards that employ cognitive radio approaches rely on geolocation database to inform the base station (BS) about the spectrum opportunities in a given region, the spectrum sensing can be used in conjunction with the database approach to enhance the reliability and increase shared spectrum access opportunities. Database information may be inaccurate due to software based propagation estimation which can lead to erroneous results in varying terrain shapes that are present in remote area scenarios. Spectrum sensing will be used also to detect other SUs at the same region. In addition, there are situ- 


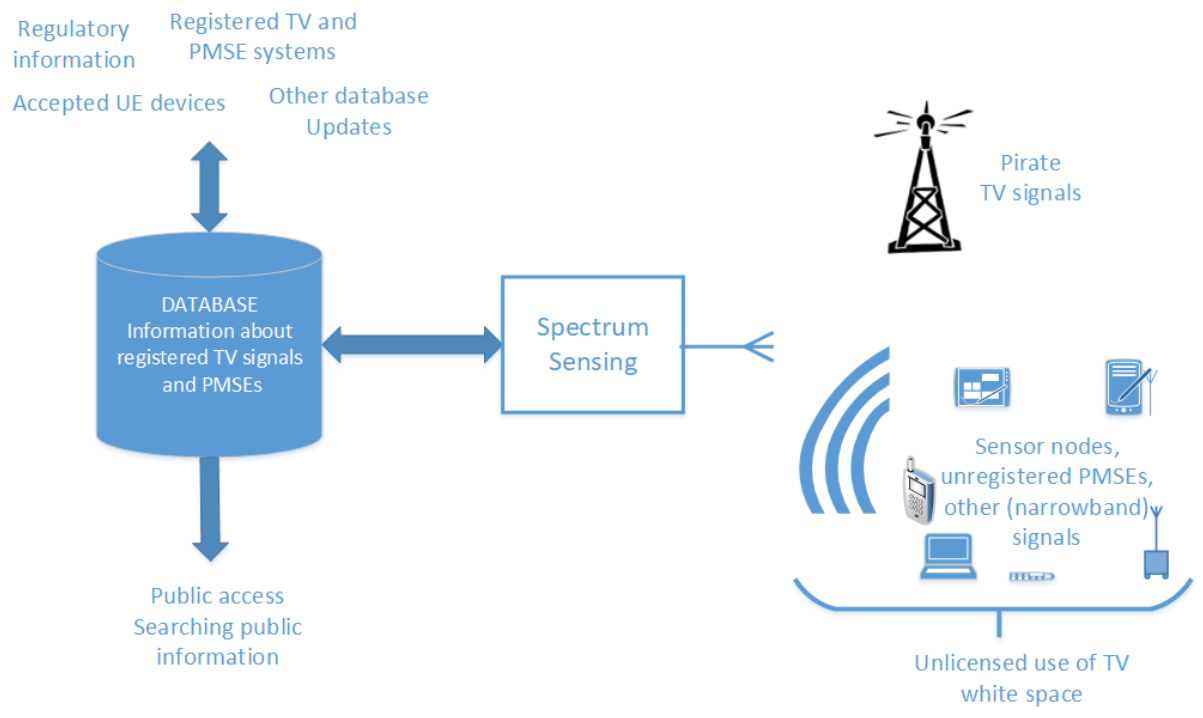

Fig. 1. System model for spectrum sensing to complement database approach.

ations where the use of spectrum sensing can provide benefits such as in the presence of unauthorized transmissions (e.g., pirate TV transmissions). Figure 1 summarizes the high-level system model for combined spectrum sensing and database approach. In the 5G-RANGE project [13], this approach is proposed to be a feasible solutions for remote area system which targets to dynamically exploit free spectrum holes available at TV bands. Next, we introduce the developed spectrum sensing method, which is considered as a one feasible option for 5G-RANGE system, in detail.

\section{Spectrum Sensing}

Here will be described the WIBA method which is considered to be used for spectrum sensing in 5G-RANGE system. In the performance evaluation, a wellknown LAD method is used as a point of comparison and will be introduced shortly in this section.

Both the methods are blind spectrum sensing algorithms that are able to iteratively estimate the noise level by using adaptive thresholds. They can be applied to a wide set of situations. The signals to be detected must be narrowband with respect to the analyzed bandwidth (BW). The narrower the signal, the better the method perform, hence it is reasonable to make an assumption that the BW has to be at most $50 \%$ of the analyzed BW $[8,10]$. According to [10], as the signal's BW gets wider, SNR must be higher in order to achieve an acceptable sensing performance. Note, that the methods can be used in any frequency band $(\mathrm{kHz}-\mathrm{GHz})$. 
Table 1. Threshold parameter values $T$ for different $P_{F A}$ and $M$ values.

\begin{tabular}{|l|l|l|l|l|}
\hline$P_{F A}$ & $M=1$ & $M=4$ & $M=10$ & $M=100$ \\
\hline 0.1 & 2.303 & 1.670 & 1.512 & 1.130 \\
0.01 & 4.605 & 2.511 & 1.878 & 1.247 \\
0.001 & 6.908 & 3.266 & 2.266 & 1.338 \\
\hline
\end{tabular}

The signal detection is based on the estimated noise level, therefore information about the noise level or present signal(s) are not needed. The noise is assumed to be a white Gaussian process. Even though the assumption is that the noise is Gaussian, it has been shown that the signal can be found even if the noise is not purely Gaussian [10]. A detection threshold is used to divide received samples into two sets: one set contains estimated noise-only samples, and another set contains estimated signal samples and noise. Threshold selection is addressed by the constant false alarm rate (CFAR) principle, which means that the used detection threshold parameter is calculated a priori using a pre-selected desired false alarm rate $P_{F A}$ and the statistical properties of the noise $[14,15]$.

In this paper, it is assumed that the samples $x_{i}$, taken in the frequencydomain are zero mean, independent Gaussian distributed (i.i.d.) complex random variables. The energy of sample $x_{i}$ is $y_{i}=\left|x_{i}\right|^{2}$, which follows a chi-squared distribution. By assuming a chi-squared distributed variables with $2 M$ degrees of freedom, the threshold parameter $T$ can be found by solving [16-18]

$$
P_{F A}=e^{-T M} \sum_{k=0}^{M-1} \frac{1}{k !}(T M)^{k},
$$

where $P_{F A}$ is the pre-selected false alarm rate. Note that (1) does not depend on the noise variance. When $M=1$, variables follow chi-squared distribution with two degrees of freedom, and 1 leads to a threshold parameter

$$
T=-\ln \left(P_{F A}\right) \text {. }
$$

Example threshold parameter values $T$ for different values of $P_{F A}$ and $M$ are presented in Table 1. For example, when $M=1$ and $P_{F A}=0.01$, then $T=4.605$. Note that the threshold parameter is constant for specific $M$ and $P_{F A}$, and can be calculated beforehand.

\subsection{WIBA Method}

In the WIBA method, overlapping is used in spectrum sampling. Assume that $N$ energy samples $y$ are obtained during the channel sensing. The observed samples are divided into $L$ overlapping blocks (i.e. detection windows) with length $M$. An example case, where the degree of overlapping between two blocks is $50 \%$, is illustrated in Figure 2. Samples in each block are summed up among themselves, 


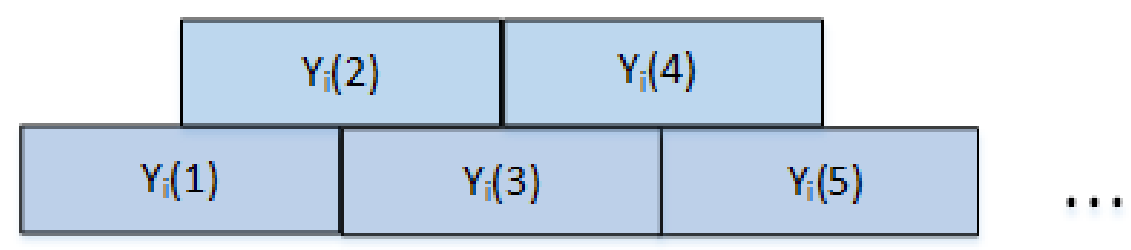

Fig. 2. Illustration of $50 \%$ overlapping when there are $L$ overlapping blocks and the length of one block is $M$.

so each block $Y_{i}(l), l=1, \cdots, M$ consists of samples $\frac{k M}{2}+1, \cdots, \frac{k M}{2}+M$, $k=0, \cdots, L-1$. The signal detection threshold is [8]

$$
T_{h}=T \frac{1}{L} \sum_{i=1}^{L} Z_{i}
$$

where $T$ comes from (1) and $Z_{i}$ is the total energy in $i$ th block, i.e., $Z_{i}=$ $\sum_{l=1}^{M} Y_{i}(l)$ when $i=1,2, \ldots, L$.

\subsection{The LAD Method}

The LAD method [9] [10] utilizes iterative forward consecutive mean excision (FCME) threshold setting process. Therein, the threshold is $T_{h}=T \bar{y}$, where threshold parameter $T$ comes from (2) and $\bar{y}$ is the mean of energy samples. Threshold setting procedure is described more detailed, e.g., in [17]. After calculating two FCME thresholds, the upper and lower ones, using two different threshold parameters, the LAD method uses clustering to group adjacent samples assumed to be from the same signal. The LAD method clusters together adjacent samples above the lower threshold. The cluster is accepted to be caused by a signal if at least one of the samples is also above the upper threshold. The performance of the LAD method can be improved using an ACC parameter that allows $p$ (usually $p=3$ ) samples to be below the lower threshold between two accepted clusters [10].

\section{Simulation Results}

In the computer simulations, the WIBA method was studied and compared to the well-studied LAD method which has been found to outperform general ED methods [9], [10], [17]. In this work the effect of the detection window length $M$ to the detection performance in different channel situations was studied. RMSE for the bandwidth estimation was evaluated, as well as detection probability over multipath channels in multi-signal situations. It was assumed an AWGN channel and the measured signal, occupying $5-30 \%$ of the channel BW, was 
Table 2. Optimal detection window lengths $M$ for signals with different bandwidths (samples / \%].

\begin{tabular}{|c|c|}
\hline Detection window length $M$ & signal BW samples / \% \\
\hline 10 samples & BW 10 samples / 1\% \\
40 samples & BW 40 samples / 4\% \\
52 samples & BW 52 samples / 5\% \\
102 samples & BW 102 samples /10\% \\
204 samples & BW 204 samples / 20\% \\
306 samples & BW 306 samples / 30\% \\
\hline
\end{tabular}

based on BPSK modulation. The BPSK signal was band-limited by a RC filter with a roll-of factor of 0.22 . The number of frequency domain samples $N=1024$. SNR was defined as a total signal power per total noise power, i.e., over $N$ samples. The probability of detection $P_{d}$ was defined so that the signal is defined to be detected if threshold is crossed at its center frequency. The amount of Monte Carlo iterations were 1000. The WIBA method used $P_{F A}=0.01,50 \%$ overlapping, $M$ varied, and $L \approx 2 \frac{N}{M}$. The used threshold parameter $T$ depend on $M$ as shown in Table 1. Detection window length $M$ was defined to be optimal when it equals to the signal bandwidth. Table 2 shows optimal detection window lengths $M$ for signals with different bandwidths. For example, window length $M=52$ samples is optimal for signal with $5 \% \mathrm{BW}$ (= 52 samples). The LAD threshold parameters were $13.81\left(P_{F A}=10^{-6}\right)$ and $2.66\left(P_{F A}=0.07\right)$ [10], and $M=1$ (=no windowing). An adjacent version of the LAD method with ACC parameter $p=3$ was used.

\subsection{One Signal Scenario}

In [8], an initial performance evaluation of WIBA was done by studying the probability of detection and the number of detected signals in one-signal case. Based on those results it was concluded that a very long window is preferred instead of the very short one when considering performance in terms of $P_{d}$.

In this paper, BW estimation accuracy is studied. Relative mean square error (or root mean squared relative error, RMSRE) of BW estimation is defined to be

$$
R M S E_{\gamma}=\sqrt{\frac{1}{N} \sum_{i=1}^{N}\left(\frac{\gamma_{i}-\hat{\gamma}_{i}}{\gamma_{i}}\right)^{2}},
$$

where $\gamma_{i}$ is the BW and $\hat{\gamma}_{i}$ is the estimated BW.

Table 3 shows the results when there is one signal with 10, 20 or $30 \% \mathrm{BW}$, and $M=52,102,204$ and 306. Results for optimal window lengths are in bold. For example, when the signal BW is $10 \%$ and $M=102$, RMSE is $100 \%$ for WIBA method. On the other hand, RMSE for LAD ACC method is only $8 \%$. It can be noticed that using WIBA method, too long window degrades the BW 
Table 3. Relative Mean Square Error (RMSE) [\%] in the one signal scenario for 10, 20 , and $30 \%$ bandwidth when $M=52,102,204$ and 306 .

\begin{tabular}{|l|r|r|r|r|r|r|}
\hline & \multicolumn{3}{|c|}{ WIBA, $M=$} & LAD ACC \\
BW \% (samples) & 52 & 102 & 204 & 306 & \\
\hline BW 10\% (102) & 58 & $\mathbf{1 0 0}$ & 300 & 500 & \\
BW 20\% (204) & 15 & 50 & $\mathbf{1 0 0}$ & 198 & 8 \\
BW 30\% (306) & 7 & 1.5 & 33 & $\mathbf{1 0 0}$ & \\
\hline
\end{tabular}

estimation accuracy because in that case, the detected signal does not cover the whole window.

In Figure 3, RMSE vs. SNR is presented for a signal occupying $10 \%$ of the overall BW (corresponding to the first line in Table 3). Figure 3 also shows at which SNR values each method achieve $P_{d}=0.9$. Note that the WIBA method has $P_{d}=0.9$ when $-13 \mathrm{~dB} \leq \mathrm{SNR} \leq-11 \mathrm{~dB}$, depending on the $M$, while the LAD ACC method achieves $P_{d}=0.9$ when $\mathrm{SNR}=5 \mathrm{~dB}$. That is, the performance difference is $16-18 \mathrm{~dB}$. Because the WIBA method is able to operate in low SNR region (SNR $<-10 \mathrm{~dB}$ ), it is feasible for remote area scenarios, where long distance propagation makes received signal's strength weak. However, the LAD method has better BW estimation accuracy. It can be seen that, for the WIBA method, RMSE rises with the SNR when $M$ is large. This is because the fact that as the detection performance of the LAD method depends on the bandwidth of the detected signal, the detection performance of the WIBA method depends also on the length of the used detection window.

\subsection{Multi-Signal Situation}

In this scenario, it is assumed that two RC-BPSK signals are present in the channel. The results are presented in Table 4, considering that there are one or two signals occupying $10 \%$ and $5 \%$ of the channel BW, respectively. For example, when $M=102$ and there are two signals with BWs corresponding to $10 \%$ and $5 \%$, the performance of the WIBA method is at most $1 \mathrm{~dB}$ worse when compared to the one signal scenario. Optimal values for $M$ are 102 for $10 \% \mathrm{BW}$ signal and 52 for $5 \%$ BW signal. Note that $M$ does not effect the LAD ACC performance because there is no windowing. Based on Table 4, multi-signal situation has only slight effect to the performance of the methods.

In Figure 4, the number of detected signals vs. SNR is presented. There are two signals with $5 \%$ and $10 \%$ BWs, and $M=10,40,52,102$ and 204. This figure also shows at which SNR each approach achieve $P_{d}=0.9$. For example, when $M=52, P_{d}=0.9$ when $\mathrm{SNR}=-12 \mathrm{~dB}$. The window is very short when $M=10$ and $M=40$. Optimal window lengths are $M=52$ for $5 \%$ BW signal and $M=102$ for $10 \%$ BW signal. When $M=40,52$ and 102, the WIBA method estimated the number of signals correctly when $P_{d}=0.9$. It can be seen that too short window $(M=10)$ estimates the number of detected signals correctly only when SNR is larger: when $P_{d}=0.9(\mathrm{SNR}=-5 \mathrm{~dB})$, the number of detected 


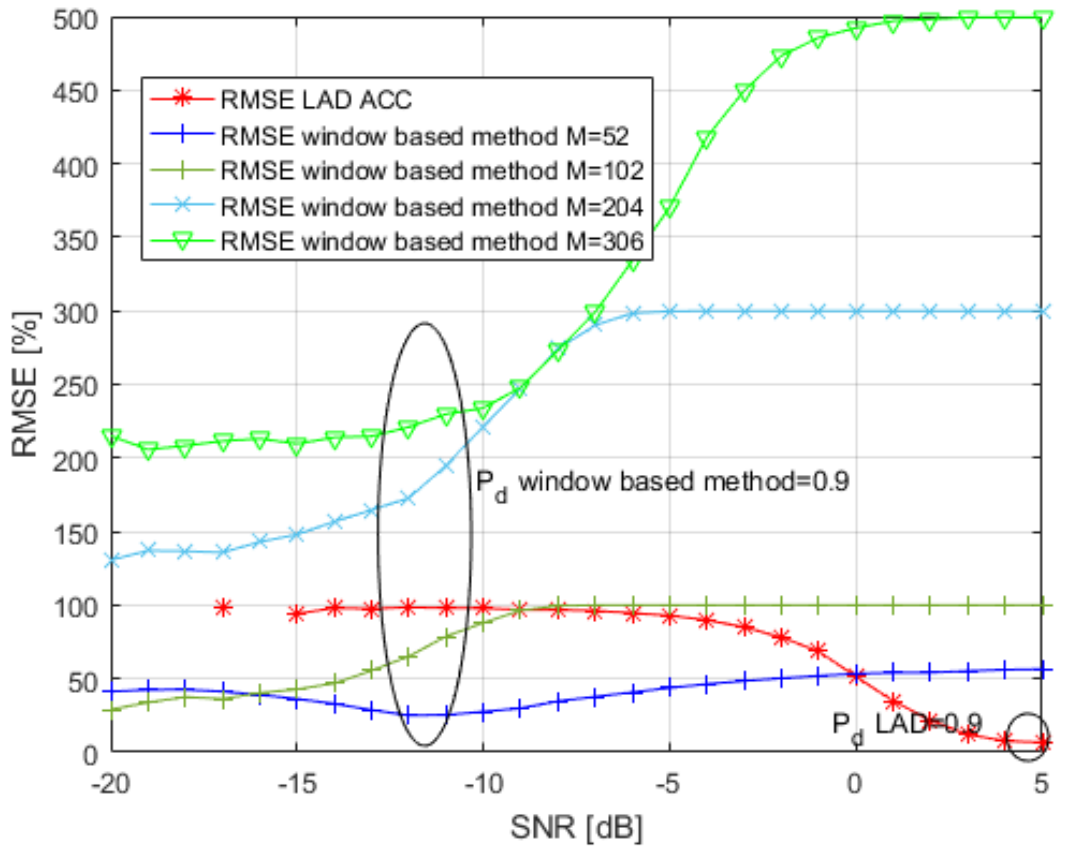

Fig. 3. RMSE vs. SNR results for the case when bandwidth of the signal is $10 \%$. 
Table 4. Required SNR $[\mathrm{dB}]$ for $P_{d}=0.9$ when there is one or two signals present.

\begin{tabular}{|c|c|c|c|r|}
\hline $\begin{array}{c}\text { Window length } \\
M\end{array}$ & $\begin{array}{c}\text { \# of } \\
\text { signals }\end{array}$ & $\begin{array}{c}\text { Signal } \\
\mathrm{BW}\end{array}$ & $\begin{array}{c}\text { WIBA } \\
\text { method } \\
P_{d}=0.9\end{array}$ & $\begin{array}{r}\text { AAD ACC } \\
\text { method } \\
P_{d}=0.9\end{array}$ \\
\hline$M=102$ & Two & $10 \%$ & $-13 \mathrm{~dB}$ & $3 \mathrm{~dB}$ \\
& & $5 \%$ & $-13 \mathrm{~dB}$ & $-1 \mathrm{~dB}$ \\
$M=102$ & One & $10 \%$ & $-13 \mathrm{~dB}$ & $1 \mathrm{~dB}$ \\
& & $5 \%$ & $-14 \mathrm{~dB}$ & $-2 \mathrm{~dB}$ \\
$M=52$ & Two & $10 \%$ & $-12 \mathrm{~dB}$ & $3 \mathrm{~dB}$ \\
& & $5 \%$ & $-14 \mathrm{~dB}$ & $-1 \mathrm{~dB}$ \\
$M=40$ & Two & $10 \%$ & $-11 \mathrm{~dB}$ & $3 \mathrm{~dB}$ \\
& & $5 \%$ & $-14 \mathrm{~dB}$ & $-1 \mathrm{~dB}$ \\
$M=10$ & Two & $10 \%$ & $-5 \mathrm{~dB}$ & $3 \mathrm{~dB}$ \\
& & $5 \%$ & $-10 \mathrm{~dB}$ & $-1 \mathrm{~dB}$ \\
$M=10$ & One & $10 \%$ & $-5 \mathrm{~dB}$ & $1 \mathrm{~dB}$ \\
& & $5 \%$ & $-11 \mathrm{~dB}$ & $-2 \mathrm{~dB}$ \\
\hline
\end{tabular}

signals is 2.7, and achieves 2 when $\mathrm{SNR}=1 \mathrm{~dB}$. This corresponds the behaviour of the LAD ACC method. When using the LAD ACC method, the number of detected signals is about 2.2 at its best. As can be seen from Figure 5, the BW estimation accuracy of the WIBA method may suffer if the window is too wide ( $M=204$, for instance). Large $M$ means that closely spaced signals can be seen as one signal by the sensing technique.

\subsection{Scenario with Multipath Channel}

Multipath channel can be a very challenging environment for spectrum sensing since it includes typically LOS and scattered components (Rician channel). Let $a_{i}, i=1, \cdots, K$ be the average amplitude of each signal component. The total energy of signal components is $E=\sum_{i=1}^{K} a_{i}^{2}$.

In the simulations, there were LOS component and two scattered components $(K=3)$. The first scattered component had energy $3 \mathrm{~dB}$ below the LOS component, while the second scattered component had energy $6 \mathrm{~dB}$ below the LOS component. Used delays were 2, 20 and 100 samples for the first scattered component, and 10,40,70 and 100 for the second scattered component.

In Figure 6, detection probability vs. SNR in multipath channel case is considered. Signal BW is $10 \%, M=102$ (optimal), and there are two multipath components with different delays in samples. It can be seen that the multipath enhances the detection performance by $1-2 \mathrm{~dB}$, regardless of the sample delays. This is because constructive summation increases the energy of the signal, and this affects the detection when using ED based methods. Here, SNR is defined to include only LOS energy. If SNR includes energy of LOS and scattered components, the performance is $1-2 \mathrm{~dB}$ worse, and the performance equals to the non-multipath performance. 


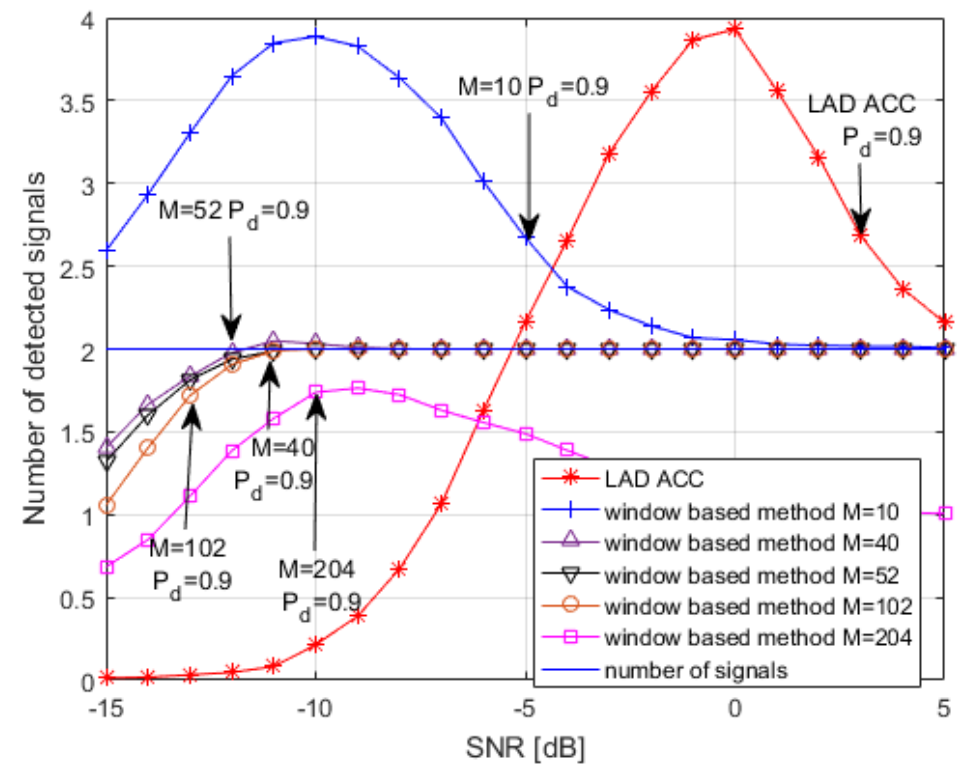

Fig. 4. Number of detected signals vs. SNR results. There are two signals with $10 \%$ and $5 \%$ bandwidths to be detected.

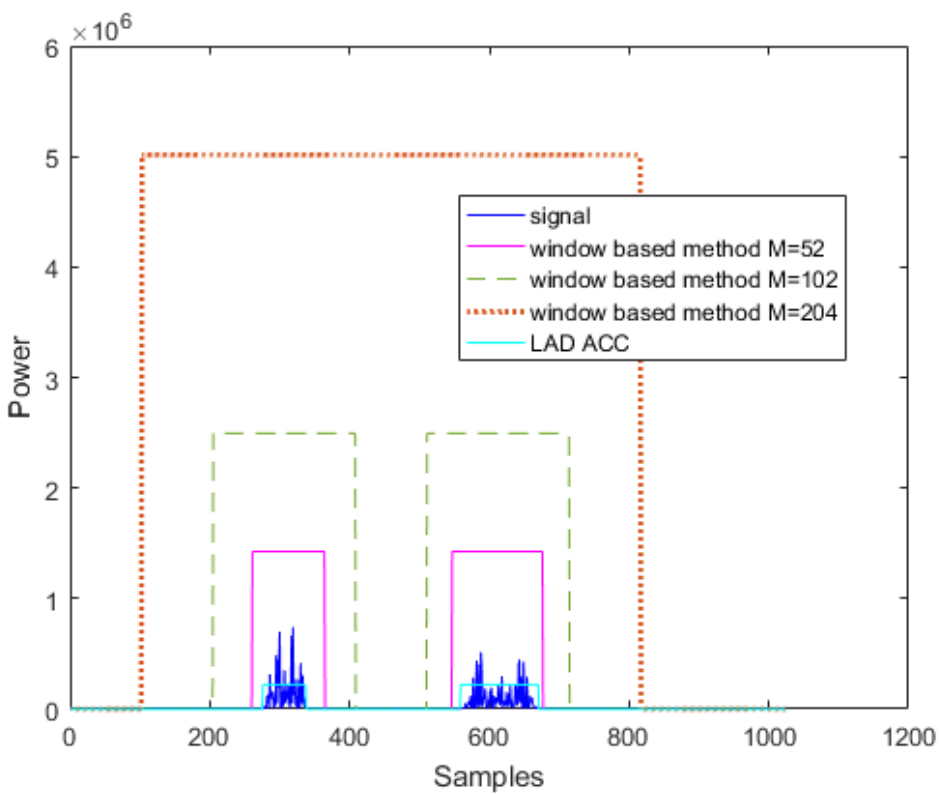

Fig. 5. One snapshot of two simulated signals with $5 \%$ and $10 \%$ bandwidth. $M=52$ (optimal for 5\% BW signal), 102 (optimal for 10\% BW signal), and 204. 


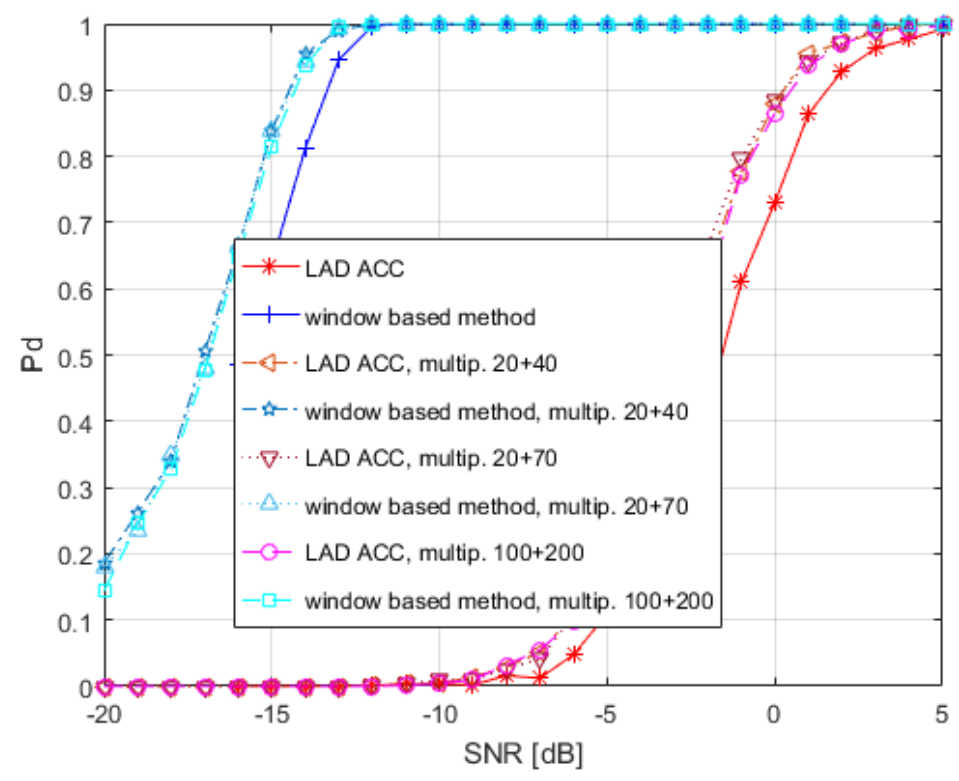

Fig. 6. Probability of detection vs. SNR in multipath channel case. Signal bandwidth is $10 \%$ and $M=102$. SNR is calculated for LOS component.

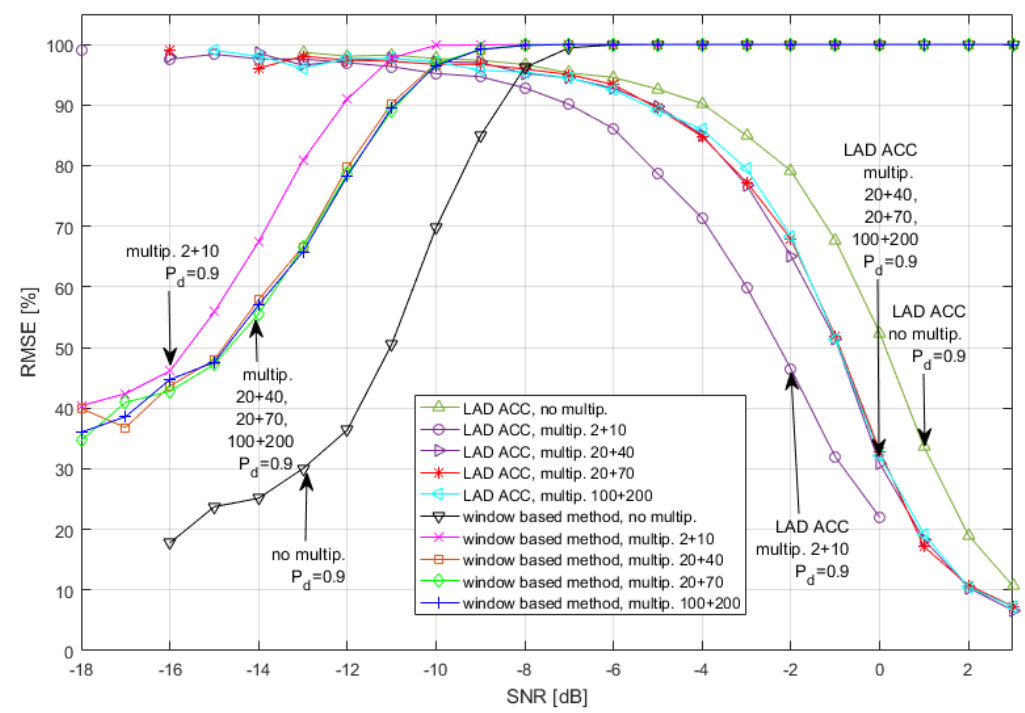

Fig. 7. RMSE vs. SNR results in the presence of multipath components. BW of the signal is $10 \%$ and $M=102$. 
Next, the bandwidth estimation accuracy is studied. In Figure 7, RMSE vs. SNR is presented in the presence of multipath. Here, signal BW is $10 \%$ of the channel bandwidth and $M=102$ (optimal). This figure also shows the minimum SNR values when the $P_{d} \geq 0.9$ is achieved. For example, when there is no multipath and the WIBA method is used, a $\mathrm{SNR}=-13 \mathrm{~dB}$ is required to achieve $P_{d}=0.9$. As a comparison, the LAD ACC method requires $\mathrm{SNR}=1$ $\mathrm{dB}$ to achieve $P_{d}=0.9$. The difference between the WIBA and the LAD ACC methods is $14 \mathrm{~dB}$. However, it can be noticed that the LAD method has better BW estimation accuracy. The multipath has about $1-3 \mathrm{~dB}$ effect to the RMSE performance.

\section{Conclusions}

Remote area connectivity problem can be solved by using lower frequencies and making shared license-free spectrum access possible to enable cost-efficient solution for low user density areas. Traditional database approach can be enhanced by including spectrum sensing to more accurately characterize the current spectrum usage in order to identify more opportunities for shared spectrum access. In this work, the performance of a spectrum windowing based energy detection method WIBA was studied, and comparison was made with the well-studied LAD ACC method. Probability of detection, relative mean square error for the bandwidth estimation, and the number of detected signals were evaluated. From the simulations results, one can conclude that the WIBA method has better detection probability than the LAD ACC method. The WIBA method is able to operate with SNR below $-10 \mathrm{~dB}$, depending on the signal and window lengths. The WIBA method is suitable for $5 \mathrm{G}$ applications especially for rural and remote areas due to its good detection performance in low SNR areas. The effect of the detection window length to the detection performance in different channel situations was also studied. Too long detection window degrades the performance of the WIBA method. The LAD ACC method outperforms the WIBA method in terms of bandwidth estimation accuracy. Therefore it can be concluded that if signal detection at a given frequency band is enough for the system, WIBA method is preferred. If BW estimation accuracy is important, LAD ACC could be used after WIBA method to improve the BW estimation.

\section{Acknowledgment}

This research has received funding from the European Union Horizon 2020 Programme (H2020/2017-2019) under grant agreement N0. 777137 and from the Ministry of Science, Technology and Innovation of Brazil through Rede Nacional de Ensino e Pesquisa (RNP) under the 4th EU-BR Coordinated Call Information and Communication Technologies through 5G-RANGE project. In addition, this research has been financially supported in part by Academy of Finland 6Genesis Flagship (grant 318927) and CNPq-Brasil. 


\section{References}

1. Bockelmann C. et al.: Towards Massive Connectivity Support for Scalable mMTC Communications in 5G Networks. In: IEEE Access, vol. 6, pp, (2018).

2. Li B., Li S., Nallanathan A., Zhao C.: Deep Sensing for Future Spectrum and Location Awareness 5G Communications. In: IEEE Journal on Selected Areas in Communications, vol. 33, num. 7, pp. 1331-1344, (2015).

3. Atat R., Liu L., Chen H., Wu J, Li H., Yi Y.: Enabling cyber-physical communication in $5 \mathrm{G}$ cellular networks: challenges, spatial spectrum sensing, and cybersecurity. In: IET Cyber-Physical Systems: Theory and Applications, col. 2, Iss. 1, pp. 49-54, (2017).

4. Ejaz W., Ibnkahla M.: Multiband Spectrum Sensing and Resource Allocation for IoT in Cognitive 5G Networks. In: IEEE Internet of Things Journal, vol. 5, no. 1, pp. 150-163, (2018).

5. Vartiainen J., Hoyhtya M., Vuohtoniemi R., Ramani V.V.: The Future of Spectrum Sensing. In: ICUFN 2016, pp. 247-252.

6. Akyildiz I.F, Lo B.F., BalakrishnanR.: Cooperative Spectrum Sensing in Cognitive Radio Networks: A survey. In: Physical Communication, vol. 4, no. 1, pp. 40-62, (2011).

7. Liu X., He D., Jia M.: 5G-based wideband cognitive radio system design with cooperative spectrum sensing. In: Physical Communications, vol. 25, part 2, pp. 539-545, (2017).

8. Saarnisaari H., Vartiainen J.: Spectrum Window Based Signal Detection at Low SNR. In: International Conference on Military Communications and Information Systems (ICMCIS) 2018, Poland.

9. Vartiainen J., Lehtomaki J.J., Saarnisaari H.: Double-threshold based narrowband signal extraction. In: IEEE Veh. Technol. Conf, pp. 1288-1292, (2005).

10. Vartiainen J.: Concentrated signal extraction using consecutive mean excision algorithms. In: Ph.D. Dissertation, Acta Univ Oul Technica C 368. Faculty of Technology, University of Oulu, Finland, (2010).

11. ECC : Technical and operational requirements for the possible operation of cognitive radio systems in the white spaces of the frequency band 470-790 MHz. In: ECC Report 159, (2011).

12. FCC : Amendment of the Commission's Rules with Regard to Commercial Operations in the 3550-3650 MHz Band. In: Report and Order, FNPRM , FCC-15-47, (2015).

13. 5G-RANGE project, http://5g-range.eu/ . Last accessed 1 Mar 2019

14. Miller K.S.: Complex Gaussian Process. Siam Review vol 11, pp. 544-567, (1969).

15. Neeser F.D., Massey J-L.: Proper complex random processes with applications to information theory'. In: IEEE Trans. on Information Theory, vol. 39, pp. 1293-1302, (1993).

16. Lehtomaki J.J., Vartiainen J., Juntti M., Saarnisaari H.: CFAR outlier detection with forward methods. In: IEEE Trans. on Signal Processing, vol. 55, pp. 4702-4706, (2003).

17. Saarnisaari H., Henttu P.: Impulse detection and rejection methods for radio systems. Mil. Commun. Conf. (MILCOM) 2003, pp. 1126-1131.

18. Proakis J.G.: Digital Communications. In: Third Edition. McGraw-Hill, Inc., (1995). 